\title{
Between vulnerability and resilience: A network analysis of fluctuations in cognitive risk and protective factors following remission from depression
}

\author{
Kristof Hoorelbeke ${ }^{a}$, Nathan Van den Bergh ${ }^{a}$, Marieke Wichers ${ }^{b}$, \& Ernst H. W. Koster ${ }^{a}$ \\ ${ }^{a}$ Department of Experimental-Clinical and Health Psychology, Ghent University, Belgium \\ ${ }^{b}$ University of Groningen, University Medical Center Groningen (UMCG), department of Psychiatry, \\ Interdisciplinary Center Psychopathology and Emotion regulation (ICPE), Groningen, the Netherlands
}

\author{
*Corresponding author: \\ Kristof Hoorelbeke \\ Department of Experimental Clinical and Health Psychology \\ Ghent University \\ Henri Dunantlaan 2 \\ B-9000 Gent, Belgium \\ +32 9-264-94-16 \\ e-mail: Kristof.Hoorelbeke@UGent.be
}

Disclaimer: Please note that this is the uncorrected Word version, we refer to the website of the publisher for the final PDF. 


\begin{abstract}
Research exploring how cognitive risk- and protective factors relate following remission from internalizing disorders suggests a central role for resilience. However, it remains unclear what constitutes resilience in this context. Furthermore, previous studies have typically relied on crosssectional data which do not allow to map the temporal dynamics of such relations. Using a seven-day experience sampling period in 85 remitted depressed patients, we examined the interplay between five transdiagnostic vulnerability- and protective factors in daily life. We present a temporal, contemporaneous, and a between-subjects network, providing an in-depth analysis of how these factors relate to daily life fluctuations in residual symptomatology. Furthermore, we test the role of positive affect as a main resilience factor. Resilience uniquely predicted all other factors over time (temporal network). Higher levels of resilience were related to less momentary use of rumination, more deployment of positive appraisal, and lower occurrence of residual symptoms (contemporaneous network). Participants scoring high on resilience mostly engaged in positive appraisal (between-subjects network). Similar structures were obtained when substituting self-reported resilience by positive affect. This highlights the importance of resilience, and in particular, positive affectivity, to cope with stressors following remission. This may be fostered by facilitating the use of positive appraisal.
\end{abstract}

Keywords: resilience, remission, emotion regulation, depression, network, cognitive vulnerability 


\section{Introduction}

The period following remission from an internalizing mental disorder (e.g., depression or anxiety) is typically a challenging period where patients see themselves confronted with a multitude of stressors such as resuming different roles while dealing with some of the consequences of their past mental illness (e.g., financial or social problems). At the same time, unfortunately, patients may still possess vulnerability factors (e.g., impaired emotion regulation, cognitive impairments) which render them more vulnerable to develop novel episodes in confrontation with stress.

Although such vulnerability processes have often been investigated within specific mental disorders, there is good reason to approach these processes from a more transdiagnostic view. Within internalizing disorders, comorbidity rates are typically high (Maser \& Cloninger, 1990). Here, it has been argued that anxiety disorders rarely exist in isolation (Kaufman \& Charney, 2000), where individuals suffering from anxiety and stress-related disorders often demonstrate (sub)clinical depressive symptoms (Gorman \& Coplan, 1996; Hirschfeld, 2001; Kessler et al., 2008; Zhou et al., 2017). For instance, generalized anxiety disorder (GAD) shows strong symptom overlap with major depressive disorder (MDD; e.g., Hettema, 2008; Zbozinek et al., 2012), resulting in comorbidity rates running up to $98 \%$ pending on the diagnostic criteria used (e.g., Brown, Marten, \& Barlow, 1995). Moreover, in a large scale longitudinal cohort study, Moffitt and colleagues (2007) showed that, among individuals developing anxiety disorders between the age of 11 and 32, 72\% reported a lifetime history of MDD. In this context, major depressive disorder (MDD) forms a well-known predictor for the occurrence of anxiety- and stressrelated complaints. As such, a population that is of particular clinical interest to gain a better understanding of how different psychological factors are implicated in - stable remission from internalizing disorders, are remitted depressed (RMD) patients. 
After overcoming a depressive episode RMD patients often show increased stress sensitivity (e.g., Admon et al., 2015; O'Hara, Armeli, Boyton, \& Tennen, 2014) and experience residual depressive symptoms (e.g., Nierenberg et al., 2010). Moreover, compared to healthy controls, RMD patients tend to report higher levels of repetitive negative thinking such as rumination (McMurrich \& Johson, 2008; Woody, McGeary, \& Gibb, 2014), a maladaptive emotion regulation strategy that is characterized by repetitive negative thinking regarding one's feelings or problems (Nolen-Hoeksema, Wisco, \& Lyubomirsky, 2008). In addition, impairments at the level of attention- and working memory processes form an important source of dysfunctioning in daily life (Millan et al., 2012), often remaining present following remission from depression (e.g., Levens \& Gotlib, 2015). These apparent 'residual complaints' reflect increased cognitive vulnerability for depression (e.g., Judd et al., 1998; Segal et al., 2006; ten Doesschate, Bockting, Koeter, \& Schene, 2010), where existing treatments show limited effects in terms of preventing occurrence of future episodes (Beshai, Dobson, Bockting, \& Quigley, 2011; Uher \& Pavlova, 2016). As such, an important question remains how these different psychological constructs - which could be deemed as transdiagnostic cognitive risk or protective factors (e.g., Eysenck \& Fajkowska, 2017; Hankin et al., 2016; Shing, Jayawickreme, \& Waugh, 2016) - interact to promote stable remission rather than recurrence of internalizing disorders.

In this context, how one responds to stressors has important implications for mental well-being. For instance, in contrast to responding to a stressor with repetitive negative thinking, use of adaptive emotion regulation strategies such as positive (re)appraisal form a well-known predictor for well-being (e.g., Hoorelbeke, Koster, Demeyer, Loeys, \& Vanderhasselt, 2016a; but see Troy, Ford, McRae, Zarolla, \& Mauss, 2017; Troy, Shallcross, \& Mauss, 2013). As such, positive appraisal has been put forward as a key resilience factor (Kalisch, Müller, \& Tüscher, 2015), with resilience referring to the ability to cope effectively upon facing adversity, connoting inner strength, competence, optimism, and flexibility (Wagnild, 2009). 
Importantly, previous research focusing on how different cognitive risk and protective factors interact following remission from depression, suggests a central role for resilience. That is, using network analysis on a cross-sectional dataset, Hoorelbeke, Marchetti, De Schryver, and Koster (2016b) modeled the interplay between cognitive complaints, deployment of positive appraisal, repetitive negative thinking (rumination), self-reported resilience, and residual symptomatology. Resilience appeared to form the main hub, taking a central role in the network, connecting each of the variables to one another. These findings suggest that resilience, as the most strongly connected variable in the network, is key to remaining in a state of remission.

Noteworthy, network analysis forms an important innovation as it allows to model how different constructs relate to one another in a data-driven manner (Borsboom \& Cramer, 2013). In particular, network models offer alternative and insightful ways of visualizing complex data (Bringmann \& Eronen, 2018). Interestingly, network models obtained from cross-sectional datasets have shown to be predictive for the course of internalizing disorders (e.g., van Borkulo et al., 2015). In addition, network models demonstrate considerable stability in terms of replication of complex network structures (e.g., Borsboom et al., 2017; Faelens, Hoorelbeke, Fried, De Raedt, \& Koster, under review; Fried et al., 2018; for recent developments regarding stability and accuracy of cross-sectional network models, see Epskamp, Borsboom, \& Fried, 2018). As such, network analysis offers a powerful tool for exploratory research, allowing model generation, which can then inform confirmatory research.

However, recent findings suggest that cross-sectional networks do not reliably reflect the temporal order by which nodes affect one another, as indicators of potential causal pathways (Bos et al., 2017). This can be overcome by creating network models of data acquired through experience sampling methodology (ESM), assessing psychological constructs repeatedly in daily life. The advantage of this 
technique is that it allows taking into account information derived from both the intra- (i.e., momentary changes) and inter-individual level.

Interestingly, recent innovations allow the application of network analysis to time series data (Bringmann et al., 2013, 2016; Epskamp, Waldorp, Mõttus, \& Borsboom, 2018). This allows to visualize the dynamic interaction between the variables of interest, fostering an intuitive understanding of the complex structure of a model (Bringmann, 2016). That is, using a Vector AutoRegressive (VAR) approach, the combination of ESM and network analysis allows to model the temporal sequence by which different variables (i.e., nodes) interact. Specifically, temporal networks depict the unique predictive value of a given node at time $t$ for all connected nodes at time $t+1$. As such, edges in these models represent unique interactions between two given nodes over time. Based on patterns of connectivity, nodes obtain a specific position in the model reflecting the centrality of a given node within a network (e.g., using the Fruchterman and Reingold's algorithm; Fruchterman \& Reingold, 1991). This approach also allows computation of contemporaneous networks which model patterns of co-occurring activity, illustrating how variables predict one another within the same momentary assessment period. Finally, betweensubjects networks can be obtained, depicting how stationary means of different persons co-vary (Epskamp, Waldorp, et al., 2018).

In light of these statistical and methodological innovations, the first aim of this study is to provide a test of the interplay between key cognitive vulnerability and protective factors in daily life following remission from depression. Combining a seven-day ESM procedure with network analysis in RMD patients, the current study allows to map the temporal order by which two protective (positive appraisal, resilience) and two risk factors (rumination, cognitive complaints) interact with one another to predict fluctuations in depressive symptomatology in daily life (temporal network). Based on our prior findings, we expect to find a central role for resilience, linking other protective- and risk factors to 
depressive symptomatology (Hoorelbeke et al., 2016b). Furthermore, after controlling for temporal effects, we will map how these risk- and protective factors relate within each assessment period (contemporaneous network), and how stationary mean levels of the included variables co-vary (between-subjects network). Together, these network models allow an in-depth analysis of how key cognitive risk- and protective factors relate to one another following remission from depression.

A second aim of this study is to shed light on what constitutes 'resilience' following remission from depression. Resilience has typically been conceptualized as an outcome, in terms of being able to cope effectively when confronted with adversity (as shown by indicators of mental well-being). As such, research exploring how key predictors of resilience (resilience factors) relate to risk factors such as repetitive negative thinking, impairments at the level of cognitive functioning and residual complaints, is crucial for increasing our understanding of the role of resilience in remission from depression. In this context, several resilience factors have been proposed, among which stress recovery, self-compassion, promotion, and positive appraisal (e.g., Kalisch et al., 2015; Trompetter, de Kleine, \& Bohlmeijer, 2017; Waugh \& Koster, 2015), most of which are related to positive affectivity as a key ingredient. Indeed, ample studies have shown that positive affect buffers against negative effects of stressors (Garland et al., 2010; Tugade \& Fredrickson, 2004; Okely, Weiss, \& Gale, 2017; Wichers et al., 2007). As such, we test the role of positive affect as a central resilience factor following remission from depression, linking key depression vulnerability and protective factors. For this purpose, as a second step, we substitute selfreported 'resilience' by 'positive affect' and observe how this affects the obtained network models. If positive affectivity indeed forms a key resilience factor in the context of remission from depression, then substituting self-reported 'resilience' by 'positive affect' should render similar network structures suggesting a central position for positive affectivity. ${ }^{1}$ 


\section{Participants}

This study is part of a cognitive training project that was pre-registered on Open Science Framework (osf.io/g2k4w). The current study reports findings from the ESM week preceding the training procedure. 191 candidate participants were screened for participation to the study. Eligibility criteria were: (a) age 23-65, (b) history of depression, (c) possess a smartphone with a data management plan, and (d) no ongoing depressive episode, report no current substance abuse or psychotic disorder. Following the screening procedure, 90 RMD patients were enrolled in the ESM phase. Three participants prematurely engaged in the cognitive training procedure during the first week of ESM and were excluded from data-analyses. Two additional participants were excluded due to response rates $<50 \%$. The remaining 85 participants responded to $83 \%$ of the signals in a timely manner. On average, participants completed the questionnaires within 7.54 minutes $(S D=7.15)$ of the signal being sent. Sample characteristics are reported in Table 1. All participants provided written informed consent. This study was conducted in compliance with the Declaration of Helsinki and was approved by the local ethical committee of Ghent University.

\section{Measures}

\section{Screening instruments.}

We used a two-step, in time separated, screening protocol to assess eligibility for participation to the study. Candidate participants first underwent a telephone screening. This contained the relevant modules of the Mini-International Neuropsychiatric Interview (MINI; Sheehan et al., 1989; Van Vliet \& De Beurs, 2007), a structured clinical interview based on the Diagnostic and Statistical Manual of Mental Disorders. Participants whom reported a history of depression but did no longer meet the criteria for an ongoing depressive episode, nor reported current substance abuse or psychosis were considered to be potentially eligible for participation to the study. As such, these participants were invited to the Faculty 
of Psychology and Educational Science of Ghent University where the mood disorders module of the MINI structured interview was (re)administered by a licensed clinical psychologist to re-asses the state of remission from depression and lifetime history of depression. Participants whom consistently met the eligibility criteria during this two-step screening protocol were enrolled in the study.

\section{Questionnaire.}

Severity of residual depressive symptomatology was assessed at baseline using the Beck Depression Inventory (BDI-II-NL; Beck, Steer, \& Brown, 1996; Van der Does, 2002). This 21-item selfreport measure with a total score ranging from 0 to 63 has demonstrated good psychometric properties. The Cronbach's Alpha obtained for the BDI-II-NL in the current study was .92.

\section{ESM measures.}

Over a period of seven days, participants received six signals a day between 9AM and 9PM. We used a time-stratified schedule where each day was divided into six intervals of 120 minutes. Within each of these intervals, signals were sent at random moments. If no response was given within the first 15 minutes, a reminder signal was sent. Signals timed-out 30 minutes after sending the first signal. The minimum distance between two scheduled signals was 30 minutes. Signals were sent as text messages using SurveySignal (Hofmann \& Patel, 2015), containing a unique link that directed participants to a LimeSurvey questionnaire.

At each signal, participants completed 13 items, among which following items are relevant for the hypotheses tested in the current study: (a) Participants rated the extent to which they were in a positive affective state using three high arousal indicators of positive affect ('energetic', 'happy', 'satisfied'; Hoorelbeke et al., 2016a). Positive affect was computed using a compound score of these items (Cronbach's Alpha $=.86)$. (b) Two items assessed the extent to which participants deployed 
rumination ('focused on feelings', 'focused on problems', Cronbach's Alpha $=.65$; Moberly \& Watkins, 2008). These items were defined to assess the extent to which participants experience repetitive negative thinking regarding either feelings or problems. (c) The extent to which participants used positive appraisal was assessed using one item ('focus on a positive meaning'; Hoorelbeke et al., 2016a), referring to the extent to which participants cognitively (re)appraised events in a positive manner. (d) Participants rated the extent to which they 'felt resilient' (resilience), referring to the extent to which participants considered themselves able to effectively cope with adversity in daily life. In addition, participants rated the extent to which they (e) 'experienced cognitive complaints' as an indicator of experienced executiveand working memory impairments. For this purpose, participants were instructed to report the extent to which they experienced difficulties in daily life related to impaired working memory or broader executive functioning (e.g., attention). Participants also reported the extent to which they (f) 'experienced depressive symptoms' in daily life, where we aimed to capture a wide spectrum of experiences relating to depression in line with Richmond et al. (2015). Participants were informed that this referred to main depressive features based on the Diagnostic and Statistical Manual of Mental Disorders, such as being in a state of sustained negative affect or experiencing anhedonic features such as loss of pleasure / interest in daily life. ${ }^{2}$

All items were rated with regards to the interval between the current signal and the previous signal the participant responded to, except for the indicators of affect. The first signal of the one-week ESM-period related to the period that had extended since waking up. All items were rated on a scale from 1 ("not at all") to 100 ("very much"). Participants first rated the affective items in random order, after which the remaining items were presented at random.

\section{Procedure}


This study is part of a broader cognitive training project (osf.io/g2k4w). Eligibility of candidate participants for participation was assessed using a telephone-interview, followed by a structured interview at Ghent University. Upon providing informed consent, participants completed the baseline assessment for the cognitive training project, which included but was not limited to a one-week ESM phase preceding the training procedure. The current study reports on the findings of the ESM-procedure. For this purpose, participants' smartphones were registered in SurveySignal, after which they received extensive instructions regarding the ESM-procedure. This included a manual providing definitions of the items used. Fluctuations in the variables of interest were assessed six times a day over a period of seven days starting from the day following registration. Participants received financial reimbursement for participation to the broader cognitive training project (€75), along with written and oral debriefing.

\section{Data-analysis}

Data analysis was conducted in $\mathrm{R}$ (version 3.3.2) on the 85 participants whom completed the ESM-phase with response rates $\geq 50 \%$. At each signal, participants provided ratings with regards to the intervals between the current signal and the previous signal the participant responded to. As such, signals that did not elicit a response are not informative for the analysis and were omitted (17\%). The presented analyses are based on a total of 2,946 unique data points with an average of 33.66 time lags per person. To test our hypotheses, we conducted two separate network analyses. First, based on the cross-sectional findings of Hoorelbeke et al. (2016b), we modeled how self-reported resilience relates to fluctuations in daily life rumination, positive appraisal, cognitive complaints, and depressive symptomatology over time. Second, to examine the role of positive affectivity as a key resilience factor following remission from depression, we substituted resilience by positive affectivity.

The network models obtained from the time series data were computed using the mIVAR package (version 0.4; Epskamp, Deserno, \& Bringmann, 2017), using a two-step multilevel VAR approach 
(Epskamp, Waldorp, et al., 2018). ${ }^{3}$ As a first step, for each of the variables in the model, performance at time $t$ is predicted by all other variables at time $t-1$, including autocorrelations for the dependent variable. Within-subject centering is used in addition to taking into account subject sample means as between-subjects predictors. As a second step, multilevel regression models are run, where for a given time $t$, residuals of one variable are predicted by all other variables at time $t$. In addition to obtaining a temporal network, this allows calculation of a contemporaneous and between-subjects network (Epskamp, Waldorp et al., 2018; for a more detailed account on the two-step multilevel VAR procedure implemented by mIVAR, see supplemental material of Epskamp, Waldorp, et al., 2018).

mIVAR calls upon the qgraph package (version 1.4.4; Epskamp, Cramer, Waldorp, Schmittmann, \& Borsboom, 2012) to plot the estimated coefficients as network models. For the contemporaneous and between-subjects network, edges between two nodes were included in the network models if at least one regression parameter of both multivariate regressions was significant (OR-rule). ${ }^{4}$ Network models were plotted using the Fruchterman and Reingold's (1991) algorithm in which all nodes are positioned in the model based on their connectivity. In particular, strongly connected nodes obtain a more central position in the model. Strength of connectivity is represented by thickness of the edges between nodes, whereas the color of the edge reflects the valence of the association (green-positive; red-negative). In addition, in the temporal networks arrows are used as edges to illustrate the direction of effects. Interpretations regarding centrality of nodes rely on visual inspection of the obtained network structures, given that standardized centrality indices are less suited for multilevel VAR-models (e.g., Bringmann et al., 2013, 2016).

\section{Results}


Running head: HOORELBEKE ET AL. REMISSION NETWORKS

Descriptive statistics and intraclass correlations (ICC) for the variables of interest are reported in Table 2. The latter suggest that considerable variance in the variables is situated at the level of the individual. Pearson correlations between the variables of interest are presented in Table 3.

\section{Resilience Networks}

The temporal network (Figure 1a) demonstrates that resilience (RES) forms a central hub in the model, uniquely predicting all other nodes in the network at time $t+1$. That is, edges in the temporal network reflect unique effects from time $t$ to time $t+1$, with the arrows being indicative for the direction of these effects. As such, the obtained temporal network suggests that resilience at time $t$ predicts subsequent less deployment of rumination (RUM) and more deployment of positive appraisal (APP). In addition, feeling resilient at time $t$ predicts lower subsequent occurrence of cognitive complaints (CC) and depressive symptomatology (DEPR). For rumination, cognitive complaints and depressive symptomatology, these associations show to be unidirectional: we observed no outgoing effects from each of these nodes to resilience while controlling for the influence of the other nodes. In contrast, the associations between resilience and positive appraisal are bidirectional, where greater use of positive appraisal also predicts increased resilience at time $t+1$. Positive appraisal was mostly used when experiencing low residual symptomatology and high resilience at the previous time point, as shown by an inhibitory pathway connecting depressive symptomatology with positive appraisal, and an excitatory pathway from resilience to positive appraisal. All nodes show a positive autocorrelation. For instance, individuals reporting high levels of depressive symptomatology at time $t$ were more likely to report depressive symptoms at time $t+1$. Similar findings were observed for use of positive appraisal, rumination, self-reported cognitive complaints, and resilience. ${ }^{5}$

The contemporaneous network (Figure 1b) suggests that - within a given measurement period participants who report feeling resilient, also report greater deployment of positive appraisal, less 
deployment of rumination, and lower occurrence of depressive symptomatology. In addition, participants who report limited use of rumination and low occurrence of depressive symptomatology during a given measurement period, report less cognitive complaints. Finally, the between-subjects network (Figure 1c) suggests that participants who on average report higher levels of positive appraisal as an emotion regulation strategy also feel more resilient. In contrast, overall higher levels of rumination were related to elevated depressive symptomatology, which was linked to cognitive complaints.

\section{Positive Affect Networks}

Similar findings are obtained when substituting self-reported resilience by positive affect $(\mathrm{PA})^{6}$ : positive affect uniquely predicted all other nodes from time $t$ to time $t+1$ (Figure 2a). The current model differs from the model obtained from the analyses including resilience in terms of an additional association between rumination and positive appraisal, and omission of the association between depressive symptomatology and positive appraisal. That is, deployment of positive appraisal is mostly predicted by prior affective state. Finally, the structure of the contemporaneous (Figure $2 \mathrm{~b}$ ) and between-subjects network (Figure 2c) including positive affect was highly similar to the network structures obtained for resilience, with the exception of an additional edge between positive affect and self-reported cognitive complaints in the contemporaneous network.

\section{Discussion}

The current study set out to model how key cognitive vulnerability- and protective factors interact to predict fluctuations in internalizing symptomatology in daily life. For this purpose, we relied on a sample of RMD patients, monitoring how indicators of emotional (dys)regulation (rumination, positive appraisal), cognitive functioning, and resilience predicted fluctuations in depressive symptomatology following remission from depression. Based on initial findings of cross-sectional network models, we hypothesized to find a central role of resilience (Hoorelbeke et al., 2016b). Indeed, 
the current findings suggest that resilience plays an important role in predicting change in protectiveand risk factors in RMD patients in daily life. Feeling resilient predicted subsequent lower occurrence of rumination, cognitive complaints, and depressive symptoms. Interestingly, deployment of positive appraisal predicted increased resilience over time, which in turn predicted further deployment of positive appraisal (temporal network). In line with this, the contemporaneous network suggests that people who reported feeling resilient within a given measurement interval, typically used more positive appraisal, less rumination as well as reported lower residual symptomatology within that interval. Here, it is interesting to note that people who report feeling more resilient are those who on average report higher deployment of positive appraisal, independent of occurrence of rumination, experienced cognitive complaints, and residual symptomatology (between-subjects network).

These findings provide evidence for central resilience frameworks (Fredrickson, 1998; Kalisch et al., 2015). Our findings support the Positive Appraisal Style Theory of Resilience (Kalisch et al., 2015), suggesting that how one appraises, interprets, and analyzes potentially threatening stimuli or situations is essential for resilience. In particular, it has been suggested that positive appraisal forms the main pathway through which other resilience factors predict well-being. Indeed, our findings suggest that positive appraisal shows a bidirectional relation with positive affect, where it has been suggested that positive affect forms both a resilience factor as well as an important mechanism of resilience (Kalisch et al., 2015; Tugade \& Fredrickson, 2004). In line with this, substituting 'resilience' by 'positive affect' did not affect the main network structures obtained from the temporal, contemporaneous, and betweensubjects models, suggesting that positive affectivity plays a key role in obtaining stable remission from depression.

Our findings have several clinical implications. The obtained network models suggest that, in order to establish stable remission from depression, interventions for RMD patients should focus on 
increasing positive affectivity as a key resilience mechanism. One way to do so would be by facilitating deployment of positive appraisal, as suggested by the temporal resilience network and the betweensubjects networks. In this context, interventions such as well-being training, emotion regulation training, and mindfulness-based cognitive therapy show clear clinical potential (e.g., Garland, Geschwind, Peeters, \& Wichers, 2015; for reviews, see Leppin et al., 2014; Waugh \& Koster, 2015). In addition, our temporal resilience network indicates that residual complaints hinder deployment of positive appraisal. As such, in line with findings suggesting that residual symptoms form an important predictor of early recurrence of depressive episodes (cf. Judd et al., 1998), obtaining full remission from depression - before discontinuation of treatment - remains an important challenge. In particular, given the importance of positive affectivity as a main resilience factor, increasing our knowledge regarding the mechanisms underlying anhedonia in depression is crucial (e.g., reward learning, approach motivation; Pizzagalli, 2014). In this context, research on the Positive Valence Systems of the Research Domain Criteria (RDoC) will be highly informative (Insel et al., 2010).

First evidence suggests that psychotherapeutic interventions aimed at increasing resilience show potential in reducing depressive, anxiety, and stress-related symptoms. For instance, in a recent study Hojjat and colleagues (2017) compared effects of eight sessions of a resiliency group training program based on Schiraldi (2011) in mothers of children with ADHD, a sample which was characterized by elevated anxiety and depression levels. Beneficial effects were reported for both depression and anxiety symptoms. In addition, participants in the training condition reported increased marital satisfaction. The effects of such experimental studies (for reviews, see Joyce et al., 2018; Leppin et al., 2014; Waugh \& Koster, 2015) are in line with our findings, suggesting a key role for resilience in maintaining psychological well-being, as shown by the observed inhibitory pathways from resilience to depressive symptomatology, cognitive complaints, and repetitive negative thinking, in addition to reciprocal reinforcing effects of positive appraisal. 
As to our knowledge, the current study is the first to present network models obtained from prospective data providing an in-depth analysis of how key risk- and protective factors relate to fluctuations in residual symptomatology following remission from depression. Here, it is noteworthy that the temporal network obtained in the current study provides a replication and major extension of the network structures previously obtained from cross-sectional data in RMD patients (Hoorelbeke et al., 2016b). Nonetheless, the current study shows certain limitations that should be taken into account in future studies. First, although our network models contain some of the key cognitive vulnerability- and protective factors in the context of depression based on the existing literature, it remains a possibility that other important variables have been overlooked. Here, it is important to note that ESM procedures are limited in terms of the amount of variables that can be repeatedly assessed in daily life in order to foster compliance with the intensive sampling procedure. Related to this, in line with previous models, resilience has been conceptualized as the extent to which participants considered themselves able to efficiently cope with adversity. Assessing resilience in daily life is a challenging endeavor, where subjective resilience ratings naturally show overlap with ratings of positive appraisal, positive affect, and depressive symptoms. Several alternative operationalizations could be considered for future studies. For instance, one could model the relation between stressors and well-being, or the rate of recovery following stressors as a proxy of resilience. Additionally, future studies could include multiple resilience factors other than positive appraisal and positive affect, such as perseverance, equanimity, meaningfulness, and being self-reliant (Wagnild, 2009). An advantage of the current operationalization of resilience is that participants rated how they consider themselves able to cope with adversity, referring to a subjective experience of 'being/feeling resilient'. Although this implicitly takes into account the presence of/and successful coping with stressors, one can also report feeling resilient in absence of direct stressors (e.g., based on prior experiences and schemes, where one considers oneself being able to cope with future adversity). This aspect of resilience potentially more closely matches the feeling of 
inner strength, competence, and optimism (Wagnild, 2009), which we believe is key in understanding the close observed relation between self-reported resilience and positive affect as a main resilience factor. Importantly, providing evidence for the validity of our items, the obtained network structures replicate the network models obtained from cross-sectional studies in which resilience and positive appraisal were assessed using well-established self-report measures (e.g., Cognitive Emotion Regulation Questionnaire and the Resilience Scale; Hoorelbeke, Marchetti, et al., 2016b). However, an important advantage of the presented assessment technique is that it offers increased ecological validity, allowing to take into account intra- and interpersonal differences in how these processes occur in daily life. Third, our findings reflect the interplay between momentary fluctuations in cognitive vulnerability- and protective factors assessed over a period of seven days. Although this is highly informative to how different risk- and protective factors relate to fluctuations in symptomatology in RMD patients over time, these data are limited in time and do not provide diagnostic information. As such, they do not allow claims regarding recurrence of depressive episodes. For this purpose, longitudinal designs including clinical interviews would be necessary. Finally, given symptom overlap and the high comorbidity rates between MDD, anxiety, and stress-related disorders, RMD patients form an interesting population to study the impact of transdiagnostic cognitive risk- and protective factors following remission from an internalizing disorder. However, we should be cautious when making generalizations of our findings beyond MDD as the current study did not include diagnostic information regarding presence of (comorbid) anxiety or stressrelated disorders.

\section{Conclusion}

Using network analysis, the current study maps how key cognitive risk- and protective factors relate to daily life fluctuations in depressive symptomatology following remission from depression. In line with influential theoretical frameworks of resilience, our findings suggest a central role for positive 
affectivity, as a key resilience factor, positively impacting cognitive risk- and protective factors over time in RMD patients. This may be fostered by stimulating the use of positive appraisal. 


\section{Acknowledgements}

This research was supported by an Applied Biomedical (TBM) grant of the Agency for Innovation through Science and Technology (IWT) of the Research Foundation-Flanders (FWO), awarded to the PrevenD project (B/14730/01). KH is a Postdoctoral Fellow of the FWO (FWO.3EO.2018.0031.01)). The design was set-up by KH, EHWK and MW. Data was collected by KH and NVDB. Students Bo Swinnen and Lazlo Van Praet provided practical assistance during the screening procedure. $\mathrm{KH}$ performed the analyses and drafted the manuscript. All authors provided feedback and agree to the final version of this manuscript. Special gratitude goes to dr. Ben De Pauw for assistance provided during the process of combining the Limesurvey and SurveySignal data. The authors declare no conflicts of interest. 


\section{References}

Admon, R., Holsen, L. M., Aizley, H., Remington, A., Whitfield-Gabrieli, S., Goldstein, J. M., \& Pizzagalli, D. A. (2015). Striatal hyper-sensitivity during stress in remitted individuals with recurrent depression. Biological Psychiatry, 78, 67 - 76. Doi: 10.1016/j.biopsych.2014.09.019

Beck, A. T., Steer, R. A., \& Brown, G. K. (1996). Manual for Beck Depression Inventory II. San Antonio, Texas: Psychological Corporation.

Beshai, S., Dobson, K. S., Bockting, C. L. H., \& Quigley (2011). Relapse and recurrence prevention in depression: Current research and future prospects. Clinical Psychology Review, 31, 1349-1360. Doi: 10.1016/j.cpr.2011.09.003

Borsboom, D., Fried, E. I., Epskamp, S., Waldorp, L. J., van Borkulo, C. D., van der Maas, J. L. J., Cramer, A. O. J. (2017). False alarm? A comprehensive reanalysis of "Evidence that psychopathology symptom networks have limited replicability" by Forbes, Wright, Markon, and Krueger (2017). Journal of Abnormal Psychology, 126, 989-999. doi: 10.1037/abn0000306.

Bos, F. M., Snippe, E., de Vos, S., Hartmann, J. A., Simons, C. J.P., van der Krieke, L., . . Wichers, M. (2017). Can we jump from cross-sectional to dynamic interpretations of networks? Implications for the network perspective in psychiatry. Psychother Psychosom, 86, 175-177. Doi: $10.1159 / 000453583$

Bringmann, L. (2016). Dynamical networks in psychology: More than a pretty picture? (Dissertation). doi: 10.13140/RG.2.2.28223.10404

Bringmann, L., \& Eronen, M. K. (2018). Don't blame the model: Reconsidering the network approach to psychopathology. Psychological Review. doi: 10.1037/rev0000108

Bringmann, L. F., Pe, M. L., Vissers, N., Ceulemans, E., Borsboom, D., Vanpaemel, W., . . Kuppens, P. (2016). Assessing temporal emotion dynamics using networks. Assessment, 23, 425-435. 
Bringmann, L. F., Vissers, N., Wichers, M., Geschwind, N., Kuppens, P., Peeters, F., . . Tuerlinckx, F. (2013). A network approach to psychopathology: New insights into clinical longitudinal data. PloS ONE, 8, e60188, 1-13. Doi:10.1371/journal.pone.0060188

Brown, T. A., Marten, P. A., \& Barlow, D. H. (1995). Discriminant validity of the symptoms constituting the DSM-III-R and DSM-IV associated symptom criterion of generalized anxiety disorder. Journal of Anxiety Disorders, 9, 317-328. doi: 10.1016/0887-6185(95)0012-D

Demeyer, I., De Lissnyder, E., Koster, E. H. W., \& De Raedt, R. (2012). Rumination mediates the relationship between impaired cognitive control for emotional information and depressive symptoms: A prospective study in remitted depressed adults. Behaviour Research and Therapy, 50, 292-297. Doi:10.1016/j.brat.2012.02.012

Epskamp, S., Borsboom, D., \& Fried, E. I. (2018). Estimating psychological networks and their accuracy: A tutorial paper. Behavior Research Methods, 50, 195-212. doi: 10.3758/s13428-017-0862-1

Epskamp, S., Cramer, A., Waldorp, L., Schmittmann, V. D., \& Borsboom, D. (2012). Qgraph: Network visualizations of relationships in psychometric data. Journal of Statistical Software, 48, 1-18. Doi: 10.18637/jss.v048.i04

Epskamp, S., Deserno, M. K., \& Bringmann, L. F. (2017). mIVAR: Multi-Level Vector Autoregression. R package version 0.4

Epskamp, S., Waldorp, L. J., Mõttus, R., \& Borsboom, D. (2018). The Gaussian Graphical Model in Crosssectional and Time-series Data. Multivariate Behavioral Research, 1-28. doi: $10.1080 / 00273171.2018 .1454823$

Eysenck, M.W., \& Fajkowska, M. (2017). Anxiety and depression: Toward overlapping and distinctive features. Cognition \& Emotion, . doi: 10.1080/02699931.2017.1330255

Faelens, L., Hoorelbeke, K., Fried, E. I., De Raedt, R., \& Koster, E. H. W. (submitted). You've been defriended: Negative influences of Facebook use through the lens of network analysis. 
Fried, E. I., Eidhof, M. B., Palic, S., Costantini, G., Huisman-van Dijk, H. M., Bockting, C. L. H., . . Karstoft, K-I. (2018). Replicability and generalizability of PTSD networks: A cross-cultural multisite study of PTSD symptoms in four trauma patient samples. Clinical Psychological Science, 6. doi: 10.17605/OSF.IO/2T7QP

Fruchterman, T., \& Reingold, E. (1991). Graph drawing by force-directed placement. Software: Practice and Experience, 21, 1129-1164.

Garland, E. L., Fredrickson, B., Kring, A. M., Johnson, D. P., Meyer, P. S., \& Penn, D. L. (2010). Upward spirals of positive emotions counter downward spirals of negativity : Insights from the broadenand-build theory and affective neuroscience on the treatment of emotion dysfunctions and deficits in psychopathology. Clinical Psychology Review, 30, 849 - 864. Doi: 10.1016/j.cpr.2010.03.002

Garland, E. L., Geschwind, N., Peeters, F., \& Wichers, M. C. (2015). Mindfulness training promotes upward spirals of positive affect and cognition: Mutlilevel and autorgressive latent trajectory modeling analyses. Frontiers in Psychology, 2, 6-15. Doi: 10.3389/fpsyg.2015.00015

Gorman, J. M., \& Coplan, J. D. (1996). Comorbidity of depression and panic disorder. J Clin Psychiatry, 57, 34-41.

Hankin, B. L., Snyder, H. R., Gulley, L. D., Schweizer, T. H., Bijttebier, P., Nelis, S., . . Vasey, M. W. (2016). Understanding comorbidity among internalizing problems: Integrating latent structural models of psychopathology and risk mechanisms. Dev Psychopathol., 28, 987-1012. doi: $10.1017 / S 09545794160000663$

Hettema, J. M. (2008). The nosologic relationship between generalized anxiety disorder and major depression. Depression \& Anxiety, 25, 300-316. doi: 10.1002/da.20491

Hirschfeld, R. M. A. (2001). The comorbidity of major depression and anxiety disorders: Recognition and management in primary care. Prim Care Companion J Clin Psychiatry, 3, 244-254. 
Hofmann, W., \& Patel, P. V. (2014). SurveySignal: A convenient solution for experience sampling research using participants' own smartphones. Social Science Computer Review, 33, 235-253. Doi: $10.1177 / 0894439314525117$

Hojjat, S.K., Ziarat, H. M., Rezaei, M., Hatami, S. E., Ghaljooghi, R., \& Khalili M. N. (2017). Effectiveness of group resiliency training on anxiety, depression, and marital satisfaction in mothers of children with attention deificit hyperactivity disorder. Journal of Family Psychotherapy, 28. doi: $10.1080 / 08975353.2017 .1294967$

Hoorelbeke, K., Koster, E. H. W., Demeyer, I., Loeys, T., \& Vanderhasselt, M. A. (2016a). Effects of cognitive control training on the dynamics of (mal)adaptive emotion regulation in daily life. Emotion, 16, 945-956. doi: 10.1037/emo0000169

Hoorelbeke, K., Marchetti, I., De Schryver, M., \& Koster, E. H. W. (2016b). The interplay between cognitive risk and resilience factors in remitted depression: A network analysis. Journal of Affective Disorders, 195, 96 - 104. doi: 10.1016/j.jad.2016.02.001

Insel, T. R., Cuthbert, B., Garvey, M., Heinssen, R., Pine, D. S., Quinn, K., . . Wang, P. (2010). Research Domain Criteria (RdoC): Toward a new classification framework for research on mental disorders. American Journal of Psychiatry, 167, 748-751. doi: 10.1176/appi.ajp.2010.09091379

Joyce, S., Shand, F., Tighe, J., Laurent, S. J., Bryant, R. A., \& Harvey, S. B. (2018). Road to resilience: A systematic review and meta-analysis of resilience training programmes and interventions. BMJ Open, 8, e017858. doi: 10.1136/bmjopen-2017-017858

Judd, L. L., Akiskal, H. S., Maser, J. D., Zeller, P. J., Endicott, J., Coryell, W., . . Keller, M. B. (1998). Major depressive disorder: A prospective study of residual subthreshold depressive symptoms as predictor of rapid relapse. Journal of Affective Disorders, 50, 97-108. 
Kalisch, R., Müller, M. B., \& Tüscher, O. (2015). A conceptual framework for the neurobiological study of resilience. Behavioral and Brain Sciences, 38, 1 - 79. doi: 10.1017/S0140525X1400082X, e0

Kaufman, J., \& Charney, D. (2000). Comorbidity of mood and anxiety disorders. Depression and Anxiety, 12, 69-76. doi: 10.1002/1520-6394(2000)12:1

Kessler, R. C., Gruber, M., Hettema, J. M., Hwang, I., Sampson, N., \& Yonkers, K. A. (2008). Comorbid major depression and generalized anxiety disorders in the national comorbidity survey follow-up. Psychological Medicine, 38, 365-374. doi: 10.1017/\$0033291707002012

Leppin, A. L.,Bora,P. R.,Tilburt, J. C., Gionfriddo, M. R., Zeballos-Palacios, C., Dulohery, M. M., . . . Montori, V. M. (2014).The efficacy of resiliency training programs: A systematic review and metaanalysis of randomized trials. PloS One, 9, e111420. Doi: 10.1371/journal.pone.0111420.

Levens, S. M., \& Gotlib, I. H. (2015). Updating emotional content in recovered depressed individuals: Evaluating deficits in emotion processing following a depressive episode. Journal of Behavior Therapy and Experimental Psychiatry, 48, 156-163. Doi: 10.1016/j.jbtep.2015.03.009

Maser, J. D., \& Cloninger, C. R. (1990). Comorbidity of mood and anxiety disorders. Washington, D.C.: American Psychiatric Association.

McMurrich, S. L., \& Johnson, S. (2008). Dispositional rumination in individuals with a depression history. Cognitive Therapy \& Research, 32, 542. Doi: 10.1007/s10608-006-9093-y

Millan, M. J., Agid, Y., Brüne, M., Bullmore, E. T., Carter, C. S., Clayton, N. S., . . Young, L. J. (2012). Cognitive dysfunction in psychiatric disorders: Characteristics, causes and the quest for improved therapy. Nature Reviews, 11, 141-168. Doi: 10.1038/nrd3628

Moberly, N. J., \& Watkins, E. R. (2008). Ruminative self-focus and negative affect: An experience sampling study. Journal of Abnormal Psychology, 117, 314-323. Doi: 10.1037/0021843X.117.2.314 
Running head: HOORELBEKE ET AL. REMISSION NETWORKS

Moffitt, T. E., Harrington, H., Caspi, A., Kim-Cohen, J., Goldberg, D., Gregory, A. M., \& Poulton, R. (2007). Depression and generalized anxiety disorder: Cumulative and sequential comorbidity in a birth cohort followed prospectively to age 32 years. Arch Gen Psychiatry, 64, 651-660. doi: 10.1001/archpsyc.64.6.651

Nelson, B., McGorry, P., Wichers, M., Wigman, J. T. W., \& Hartmann, J. A. (2017). Moving from static to dynamic models of the onset of mental disorder: A review. JAMA Psychiatry, 74, 528 - 534. doi: 10.1001/jamapsychiatry.2017.0001

Nierenberg, A. A., Husain, M. M., Trivedi, M. H., Fava, M., Warden, D., Wisniewski, S. R., . . Rush A. J. (2010). Residual symptoms after remission of major depressive disorder with citalopram and risk of relapse: A STAR*D report. Psychological Medicine, 40, 41-50. Doi: $10.1017 / \mathrm{S} 0033291709006011$

Nolen-Hoeksema, S., Wisco, B. E., \& Lyubomirsky, S. (2008). Rethinking rumination. Perspectives on Psychological Science, 3, 400-424. Doi : 10.1111/j.1745-6924.2008.00088.x

O’Hara, R. E., Armeli, S., Boynton, M. H.,Tennen, H. (2014). Emotional stress-reactivity and positive affect among college students: the role of depression history. Emotion, 14, 193-202.

Okely, J. A., Weiss, A., \& Gale, C. R. (2017). The interaction between stress and positive affect in predicting mortality. Journal of Psychosomatic Research, 100, 53 - 60. Doi: 10.1016/j.jpsychores.2017.07.005

Pizzagalli, D. A. (2014). Depression, stress, and anhedonia: Toward a synthesis and integrated model. Annual Review of Clinical Psychology, 10, 393 - 423. Doi: 10.1146/annurev-clinpsy-050212185606

Richmond, S. J., Keding, A., Hover, M., Gabe, R., Cross, B., Torgerson, D., \& MacPherson, H. (2015). Feasibility, acceptability and validity of SMS text messaging for measuring change in depression during a randomized controlled trial. BMC Psychiatry, 15. doi: 10.1186/s12888-015-0456-3 
Schiraldi, G. R. (2011). The complete guide to resilience: Why it matters how to build it and maintain it. New York, NY: Resilience Training International.

Segal, Z. V., Kennedy, S., Gemar, M., Hood, K., Pedersen, R., \& Buis, T. (2006). Cognitive reactivity to sad mood provocation and the prediction of depressive relapse. Arch Gen Psychiatry, 63, $749-755$. Doi: 10.1001/archpsyc.63.7.749

Sheehan, D. V., Lecrubier, Y., Sheehan, K. H., Amorim, P., Janavs, J., Weiller, E., . . Dunbar, G. C. (1989). The Mini-International Neuropsychiatric Interview (MINI): The development and validation of a structured diagnostic psychiatric interview for DSM-IV and ICD-10. J Clin Psychiatry, 59, 22 - 33.

Shing, E. Z., Jayawickreme, E., \& Waugh, C. E. (2016). Contextual positive coping as a factor contributing to resilience after disasters. J Clin Psychol, 72, 1287-1306. doi: 10.1002/jclp.22327

Ten Doesschate, M. C., Bockting, C. L. H., Koeter, M. W. J., \& Schene, A. H. (2010). Prediction of recurrence in recurrent depression: A 5.5-year prospective study. Journal of Clinical Psychiatry, 71, 984-991. Doi: 10.4088/JCP.08m04858blu

Trompetter, H. R., de Kleine, E., \& Bohlmeijer, E. T. (2017). Why does positive mental health buffer against psychopathology? An exploratory study on self-compassion as a resilience mechanismn and adaptive emotion regulation strategy. Cognitive Therapy and Research, 41, $459-468$.

Troy, A. S., Ford, B. Q., McRae, K., Zarolla, P., \& Mauss, I. (2017). Change the things you can: Emotion regulation is more beneficial for people from lower than from higher socioeconomic status. Emotion, 17, 141-154. doi: 10.1037/emo00000210

Troy, A. S., Shallcross, A. J., \& Mauss, I. B. (2013). A person-by-situation approach to emotion regulation: Cognitive reappraisal can either help or hurt, depending on the context. Psychological Science, 24, 2505-2514. doi: 10.1177/0956797613496434

Tugade, M. M., \& Fredrickson, B. (2004). Resilient individuals use positive emotions to bounce back from negative emotional experiences. J Pers Soc Psychol, 86, 320 - 333. Doi: 10.1037/0022- 
3514.86.2.320

Uher, R., \& Pavlova, B. (2016). Long-term effects of depression treatment. The Lancet Psychiatry, 3, 9596.

van Borkulo, C., Boschloo, L., Borsboom, D., Penninx, B. W. J. H., Waldorp, L. J., \& Shoevers, R. A. (2015). Association of symptom network structure with the course of depression. JAMA Psychiatry, 72, 1219 - 1226. doi: 10.1001/jamapsychiatry.2015.2079

Van der Does, A. J. W. (2002). BDI-II-NL. Handleiding: De Nederlandse versie van de Beck Depression Inventory - 2nd edition. Lisse: Harcourt Test Publishers.

Van Vliet, I. M., \& De Beurs, E. (2007). Het Mini Internationaal Neuropsychiatrisch Interview (MINI): Een kort gestructureerd diagnostisch psychiatrisch interview voor DSM-IV en ICD-10-stoornissen. Tijdschrift voor Psychiatrie, 49, 393 - 397.

Wagnild,R. N. (2009). A review of the resilience scale. J. Nurs. Meas., 17, 105-113.

Waugh, C. E., Koster, E. H. W. (2015). A resilience framework for promoting stable remission from depression. Clin. Psychol. Rev., 41, 49 - 60. doi: 10.1016/j.cpr.2014.05.004.

Wichers, M. C., Myin-Germeys, I., Jacobs, N., Peeters, F., Kenis, G., Derom, G., . . van Os, J. (2007). Evidence that moment-to-moment variation in positive emotions buffer genetic risk for depression: A momentary assessment twin study. Acta Psychiatr Scand, 115, 451 - 457. doi: 10.111/j.1600-0447.2006.00924.x

Woody, M. L., McGeary, J. E., \& Gibb, B. E. (2014). Brooding rumination and heart rate variability in women at high and low risk for depression: Group differences and moderation by COMT genotype. Journal of Abnormal Psychology, 123, 61 - 67. doi: 10.1037/a0035450

Zbozinek, T. D., Rose, R. D., Wolitzky-Taylor, K. B., Sherbourne, C., Sullivan, G., Stein, M. B., ... Craske, M. G. (2012). Diagnostic overlap of generalized anxiety disorder and major depressive disorder in a primary care sample. Depression and Anxiety, 29, 1065-1071. doi: 10.1002/da.22026 
Zhou, Y., Cao, Z., Yang, M., Xi, X., Guo, Y., Fang, M., . . Du, Y. (2017). Comorbid generalized anxiety disorder and its association with quality of life in patients with major depressive disorder. Sci. Rep., 7, 40511. doi: 10.1038/srep40511 


\section{Footnotes:}

(1) Note that in our paper positive affect is conceptualized as a resilience factor. This conceptualization is based on research indicating that positive affect is a predictor of resilience. However, it is noteworthy that in many definitions of resilience, positive affect is also a constituent of resilience. For instance, in the operationalization of Wagnild (2009), resilience connotes inner strength, competence, flexibility, optimism, and the ability to effectively cope with adversity. This conceptual state of affairs allows the possibility that positive affect is both a predictor and indicator of resilience, with there being large overlap between resilience and positive affect. At the same time, it is possible that there is unique variance in the concept of resilience, which is based on the response to negative events, that is not fully covered by positive affect.

(2) The study reported in the current manuscript was part of the baseline phase of a pre-registered cognitive control training project (osf.io/g2k4w). This overarching project contained ESM, an experimental manipulation, in addition to a diary procedure for which the ESM-items were used with regards to the past week. The baseline assessment of this diary procedure was coupled with an assessment of the constructs of interest using well-validated self-report measures: depressive symptomatology (Beck Depression Inventory II [BDI-II], Remission of Depression Questionnaire [RDQ]), rumination (Ruminative Response Scale [RRS]), positive appraisal (Cognitive Emotion Regulation Questionnaire [CERQ]), cognitive complaints (Behavior Rating Inventory of Executive Functioning [BRIEF-A]) and resilience (Resilience Scale [RS]). This allows to validate the items used in the ESM- and diary procedure, by mapping the correlations between the baseline assessment for the diary phase and the questionnaires. Importantly, for each of the items presented in the current paper, this yielded moderate associations between the validated self- 
report questionnaire(s) of interest and the corresponding item in the 85 participants that completed the study presented in this manuscript.

(3) Multilevel VAR models consist of multiple univariate multilevel regression models, which allow taking into account information at the level of the subject and group (Bringmann et al., 2013).

(4) With the exception of one additional association in the between-subjects network, this did not yield different findings compared to the more conservative AND-rule, suggesting the obtained models are sparse.

(5) Participants continuously rated the interval between the current signal and the previous signal the participant responded to. Although this comes at the advantage of covering the full course of the seven-day ESM-procedure for each of the participants - including self-reported depressive complaints occurring overnight (e.g., waking up early, ruminating) and daytime situations during which participants failed to respond to the signal in a timely manner - this assessment method also induces additional variability in interval length. This may result in an underestimation of the edges presented in the temporal network (e.g., the predictive value of two concurrent ratings pertaining to longer intervals is likely to be smaller than for ratings that follow closer to one another in time). To control for this, additional multivariate VAR models in line with the Bringmann et al. (2013) approach were conducted using mIVARO, which allows to include interval length as a covariate. Importantly, in line with the above presented temporal network, the obtained network model provided support for the key role of resilience. However, we used the two-step multilevel analysis approach for the main results presented in this manuscript as they allow to plot the contemporaneous and between-subjects networks in addition to the temporal network, providing a more detailed understanding of the mechanisms at work.

(6) To allow visual comparison between the Resilience Networks and the Positive Affect Networks, edges of all models were scaled in relation to the highest value detected for each type of model. 
This resulted in maximum values of $.28, .46$, and .75 for the temporal, contemporaneous, and between-subjects networks respectively. For each of these models, the highest value was observed in the Positive Affect Networks. 
Table 1. Sample characteristics

RMD patients $(n=85)$

Mean age $(S D)$

$45.53(13.07)$

Gender (male : female)

$32: 53$

Residual depressive symptoms (BDI-II; SD)

$12.13(9.29)$

Mean self-reported amount of depressive episodes (SD)

$5.28(8.28)$

Mean self-reported episode length in months $(S D)$

$6.86(9.16)$

Mean time since previous episode in months (SD)

$30.14(42.50)$

$\%$ of RMDs reporting a history of multiple depressive episodes

$82 \%$

$\%$ reporting depressive episodes within a bipolar disorder

$7 \%$

$\%$ reporting current use of antidepressant medication

$51 \%$

$\%$ reporting current use of lithium

$6 \%$

$\%$ reporting current use of antipsychotic or neuroleptic medication

$2 \%$

$\%$ reporting current use of benzodiazepines

$19 \%$

$\%$ reporting history of psychotherapeutic treatment for depression

$26 \%$ 
Running head: HOORELBEKE ET AL. REMISSION NETWORKS

Table 2. Descriptive statistics

\begin{tabular}{lrrrrr}
\hline \multicolumn{1}{c}{ Variable } & $M$ & $S D$ & $Q 1$ & $Q 3$ & ICC \\
\hline Rumination & 29.07 & 16.43 & 10.00 & 45.00 & .44 \\
Positive appraisal & 53.83 & 17.06 & 40.00 & 70.00 & .53 \\
Cognitive complaints & 21.57 & 16.47 & 2.00 & 30.00 & .48 \\
Depressive complaints & 13.78 & 13.57 & 1.00 & 20.00 & .48 \\
Resilience & 50.26 & 15.14 & 35.00 & 70.00 & .53 \\
Positive affect & 56.05 & 13.58 & 43.00 & 70.00 & .49 \\
\hline
\end{tabular}

Note: SDs refer to within-subject SDs 
Table 3. Pearson correlations

\begin{tabular}{lcccccc}
\hline \multicolumn{1}{c}{ Variable } & {$[1]$} & {$[2]$} & {$[3]$} & {$[4]$} & [5] & [6] \\
\hline [1] Rumination & - & -.04 & .27 & .47 & -.07 & -.14 \\
[2] Positive appraisal & -.04 & - & -.11 & -.26 & .64 & .67 \\
[3] Cognitive complaints & .27 & -.11 & - & .30 & -.15 & -.17 \\
[4] Depressive complaints & .47 & -.26 & .30 & - & -.30 & -.35 \\
[5] Resilience & -.07 & .64 & -.15 & -.30 & - & .76 \\
[6] Positive affect & -.14 & .67 & -.17 & -.35 & .76 & - \\
\hline
\end{tabular}

Note: Except for the association between rumination and positive appraisal $(p=.06)$, all $p s<.001$. 
(A)

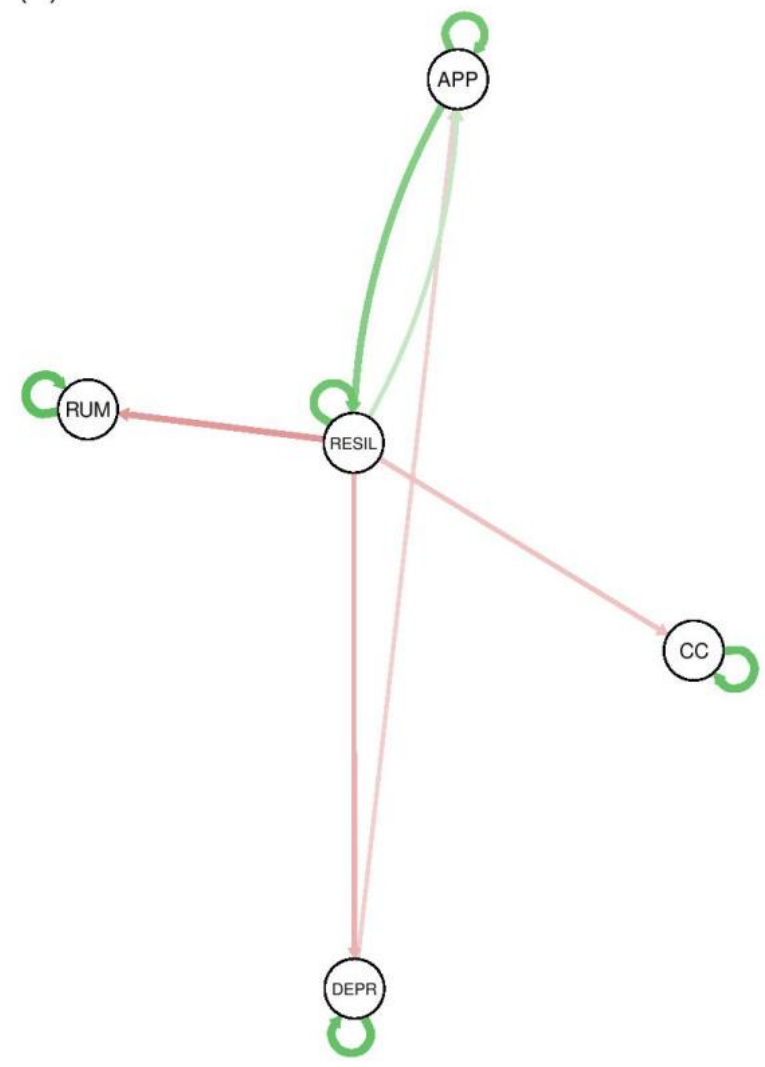

(B)

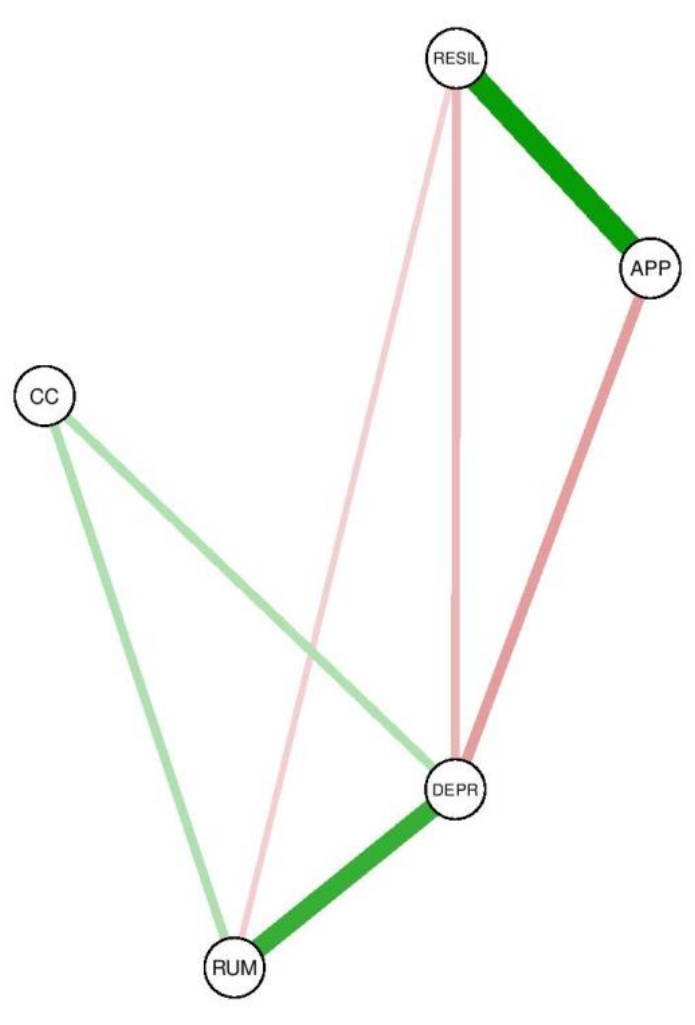

(C)

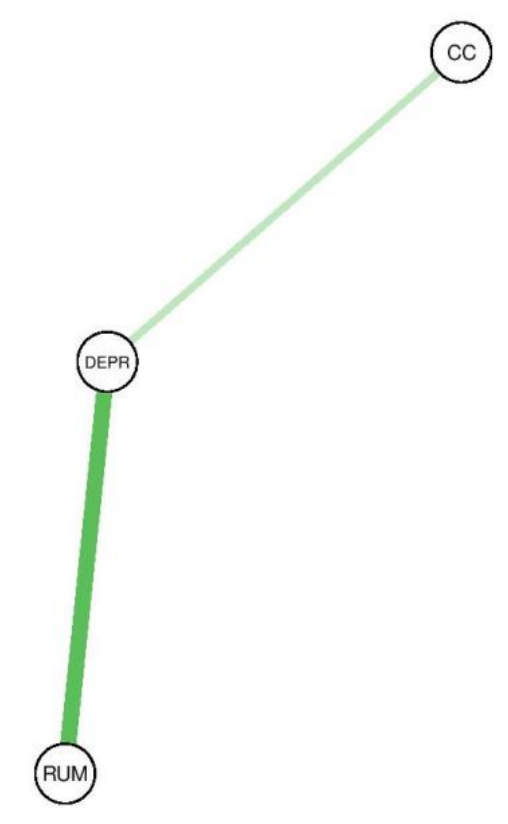

Figure 1. Resilience Networks: (A) Temporal network; (B) Contemporaneous network; (C) Between-subjects network. Note: RESIL = Resilience, RUM = Rumination, APP = Positive Appraisal, $C C=$ Cognitive complaints, $D E P R=$ Depressive symptoms. 
(A)

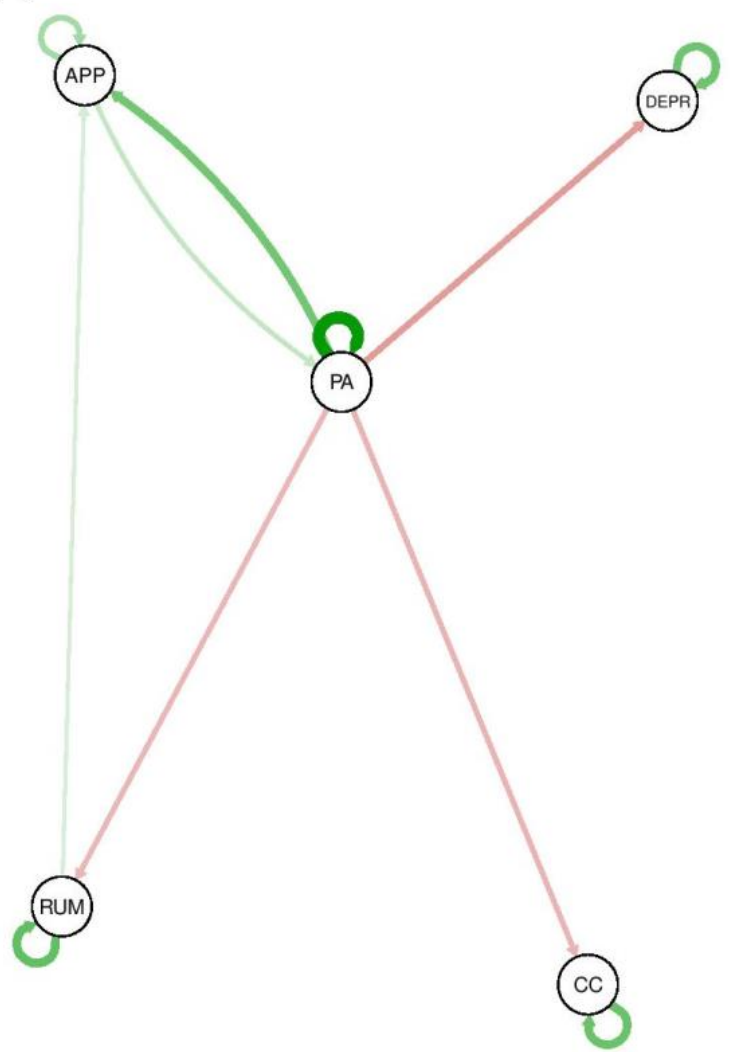

(B)

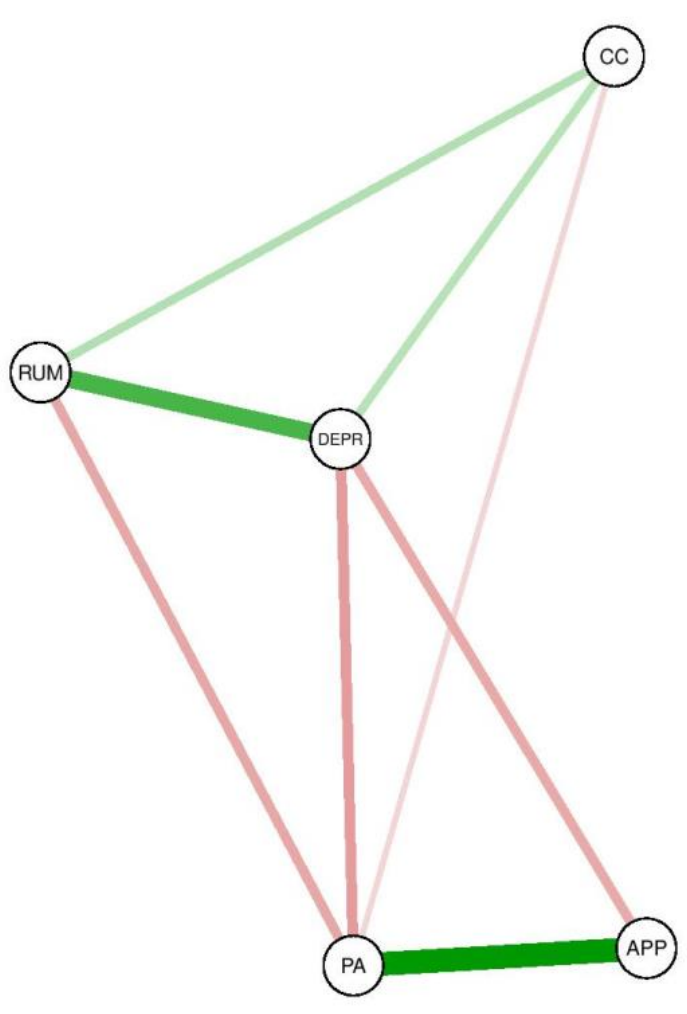

(C)

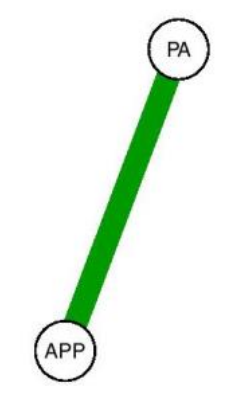

Figure 2. Positive Affect Networks: (A) Temporal network; (B) Contemporaneous network; (C) Between-subjects network. Note: PA = Positive Affect, $\mathrm{RUM}=$ Rumination, $\mathrm{APP}=$ Positive Appraisal, $\mathrm{CC}=$ Cognitive complaints, $\mathrm{DEPR}=$ Depressive symptoms. 